\title{
26348 - OUTCOME AFTER GENERAL ANESTHESIA FOR AMBULATORY SURGERY IN THE ELDERLY
}

\author{
Nicole Assmann FRCA, Sazzadul Islam, MSc; Frances Chung, FRCPC; \\ Toronto Western Hospital, UHN, Toronto, ONTARIO, Canada
}

\section{INTRODUCTION}

Very few studies have specifically investigated outcomes of the elderly after day surgery. Those that do, included large numbers of procedures carried out under local anaesthesia. Increasing numbers of elderly patients undergo complex procedures under general anesthesia (GA). This study investigated the incidence of complications in these elderly day surgery patients.

METHODS

With institutional ethics approval, the computerized medical records of all patients $\quad ; 65$ years of age, who underwent day surgery at our hospital from 2002 to 2004, were analyzed. Primary outcomes were the rates of the following procedure-related adverse events: unanticipated admission, readmission, and Emergency Room (ER) visit within 30 days.

RESULTS

1005 elderly patients (mean age 72.1, range 65 - 93) underwent surgery under GA during the 3-year period. In total, 88 patients (8.8\%) experienced an adverse event, most commonly from surgical causes (59\%). The overall incidence of unanticipated admission was $3.6 \%$. The readmission rate was $0.9 \%$, while $4.4 \%$ visited the ER within 30 days. Two $(0.2 \%)$ patients requiring readmission died from complications of their initial surgical procedure.

Patients with ASA class III had a significantly higher risk of adverse events compared to those with ASA class I or II ( $\mathrm{p} \quad 0.01$ and $\mathrm{p} \quad 0.025$, respectively). The incidence of adverse events for general surgery $(14.9 \%)$ and urology $(13.6 \%)$ was significantly higher than for orthopedic surgery $(5.4 \%, \mathrm{p} \quad 0.01)$. We identified no major anesthetic or medical morbidity after discharge.

\section{DISCUSSION}

Day surgery in the elderly can be carried out safely under GA, but the overall incidence of complications was not inconsiderable. The rates of adverse events found in this study are higher than those published for unselected adult ambulatory surgery populations 1,2. Most complications were surgical in nature, and General surgery and urology were associated with the highest risk. ASA class III was also associated with an increased risk of complications. Ambulatory surgery units with high numbers of elderly patients undergoing GA may experience higher rates of unanticipated admissions than expected. REFERENCES

1. Can J Anesth 1998;48: 612-9

2. Ann surg 1999;230:721-27 